\title{
DaMONTMATO MATHEMTCA
}

nes $2 \mathbf{I}$

14

ans

Pawel Urban

\section{CONTINUOIS SOLUTIONS OF THE FUNCTIONAL BQUATION $f\left(x^{k}(y)+y^{d}(x)\right)=f(x) f(y)$}

\section{Introduction}

Te shall consider the functional equation

$$
f\left(x f^{k}(y)+y f^{l}(x)\right)=f(x) f(y),
$$

where $k$ and $I$ belong to the set of non-negative integers $\left(f^{k}(x):=(f(x))^{k}\right)$ and $f$ is the unknown function defined in the set $R$ of all real numbers and assuming values from the set $R_{0}$ of all real numbers different from zero. In this peper we will find the general solution of (1) (for $k \neq 1$ ) in the class of continuous functions.

The equation (1) is gereralization of tho equation

$$
f\left(x f(y)+y f^{k}(x)\right)=f(x) f(y)
$$

which appeared in work [4] $(p, 18)$ at determination of certain subsemigroups of the differential group $I_{2}^{1}$, and of the equation

$$
f(x+y f(x))=f(x) f(y)
$$

which S.Goxąb and A.Schinzel obtained in tho paper [2] while searching for three-paraueter subgroups of centre-effinitj transformations group of a plane.

In particular the paper [3] contains tine construction of the general solution of the equation (2) in tis class of functions $i: \Xi-\Phi$, where $Z$ is a vactor space over a fiold $\phi$. 
The research on the oquation (2), was aleo done in papers $[1],[3],[8]$.

\section{Results}

In this section $k$.and 1 will aignify positive integers. Some property of solutions of the equetion (1) may be found in $[5],[6],[7]$. In $[7]$ we proved the following theorem.

Th $\theta \mathrm{r} \in \mathrm{m}$. The equation (1) in the class of continuous functions $f: R-R_{0}$ and such that there exists $x_{0} \neq 0$ for which $f\left(x_{0}\right)=1$, has the uniche soiution $f(x)=1$.

From the above theorem and frow the fact that $f(0)=1$ Implies what follows.

C $\circ$ r 0.1 a.r.. Let $f$ be a continuous solution of $(1)$. Now, the following cases are possible:

I.

$$
\begin{array}{r}
f(x)>1, \text { for } x<0, \\
0<f(x)<1, \text { for } x>0 \text {, }
\end{array}
$$

$$
f(0)=1 \text {; }
$$

II.

$$
0<f(x)<1 \text {, for } x<0 \text {, }
$$$$
f^{\prime}(x)>1, \text { fer } x>0 \text {, }
$$

III.

$$
\begin{aligned}
& f(0)=1 ; \\
& f(x)>1, \text { for } x \neq 0,
\end{aligned}
$$$$
f(0)=1 \text {; }
$$

IV.

$$
\begin{aligned}
0<f(x) & <1, \text { for } x \neq 0 \text { : } \\
f(0) & =1 ;
\end{aligned}
$$

v. $\quad f(x)=1$, for all $x \in R$.

Let us suppose that the continuous function $f \cdot$ satisfies the equation (1). From the form of the equation (1) It follows that: if $f((-\infty, 0)) \subset(1, \infty)$ or $f((0, \infty)) \subset(1, \infty)$, then for every $x, y \in(-\infty, 0)$ or respectively $x, y \in(0, \infty)$

$$
f\left(x f^{k}(y)+y f^{l}(x)\right)>f(x)
$$

but, if $f((-\infty, 0)) \subset(0,1)$ or $f((0, \infty)) \subset(0,1)$, then for every $x, y \in(-\infty, 0)$ or $x, y \in(0, \infty)$

$$
f\left(x f^{k}(y)+y f^{l}(x)\right)<f(x) \text {. }
$$


For $x>0$ we have

$$
\lim _{y \rightarrow \infty}\left(x f^{k}(y)+y f^{l}(x)\right)=\infty .
$$

however, ifor $x<0$, we obtain

$$
\lim _{y \rightarrow-\infty}\left(x f^{k}(y)+z f^{l}(x) ;=-\infty .\right.
$$

From continuity of the function $f$ we also have

$$
\lim _{y \rightarrow 0}\left(x f^{k}(y)+y f^{l}(x)\right)=x f^{k}(0)=x .
$$

Firom the last three relatjons and from Darhoux's property for the function $y-x f^{k}(y)+y f^{l}(x)$, we get

$$
\forall x>0 \quad \forall y>x \quad \exists y_{1}>0 \quad y=x f^{k}\left(y_{1}\right)+y_{1} f^{l}(x)
$$

and

$$
\forall x<0 \quad \forall y<x \quad \exists g_{2}<0 \quad y=x f^{k}\left(y_{2}\right)+g_{2} f^{I}(x) .
$$

(3) and $(6)$ imply that if $f((0, \infty)) \subset(1, \infty)$, then $f$ is strictly increasing function in interval $(c, \infty),(3)$ and (7) imply that if $f((-\infty, 0)) \subset(1, \infty)$, then $f$ is atrictly deoressing function in interval $(-\infty, 0) .(4)$ and $(7)$ imply that if $f((-\infty, 0)) \subset(0,1)$, than $f$ is etrictig increasing in interval $(-\infty, 0)$. At last (4) and (6) imply that if $f((0, \infty)) \subset$ $c(0,1)$, then $\rho$ is etrictly decreasing function in interval $(0, \infty)$

Hence, the function $f$ is invertible in erch of these sets: $(-\infty, 0)$ and $(0, \infty)$.

Let us assume that $f((0, \infty)) \subset(1, \infty)$; then $f_{1}:=f \mid(0, \infty)$. For the function $f_{1}$, the equetion (1) has a form

$$
f_{1}\left(x f_{1}^{k}(y)+y f_{1}^{l}(x)\right)=f_{1}(x) f_{1}(y) \text {. }
$$

Ietting $a:=f_{1}(x), b:=f_{1}(g)$, from the invertibility of $f_{1}$ we obtatn 
(8)

$$
f_{1}^{-1}(a) b^{k}+f_{1}^{-i}(s) a^{1}=f_{1}^{-1}(a b) .
$$

From the right-hand aide symatry of (8) we have

$$
f_{1}^{-1}(a) b^{k}+f_{1}^{-1}(b) a^{i}=f_{1}^{-1}(b) a^{k}+f_{1}^{-1}(a) b^{I} .
$$

If we $f i x b \neq 1$, then under assumption $k \neq 1$ we get

$$
f_{1}^{-1}(a)=\frac{f_{1}^{-1}(b)}{b^{k}-b^{1}}\left(a^{k}-a^{I}\right) \text {. }
$$

Hence

$$
f_{1}^{-1}(a)=2\left(a^{k}-a^{2}\right),
$$

where $a \in(1, \infty)$ end if $k>1$, then $p$ is a certain positive number, however if $k<1$, then $p$ is a certain negative number. It is easy to verify that functions in the form (9) for arbitrary $p>0$ or $p<0$ satisfy the equation $(8)$.

Alike at assumption $f((-\infty, 0)) \subset(1, \infty)$ for the function $f_{2}:=\left.f\right|_{(-\infty, 0)}$, we obtain

$$
f_{2}^{-1}(a)=q\left(a^{k}-a^{1}\right),
$$

where $a \in(1, \infty)$ and if $k>1$, then $q$ is a certain positive number, but if $k<1$, then $q$ is a certain negative number. get

Likewise, if $f((-\infty, 0)) \subset(0,1)$ for $f_{3}:=\left.f\right|_{(-\infty, 0 \text {, we }}$

$$
f_{3}^{-1}(a)=r\left(a^{k}-a^{l}\right)
$$

where $a \in(0,1)$ and if $k>1$, then $r$ is a certain positive number, but if $k<1$, then $r$ is a certain negative number. However, from (11) it follows that $f_{3}^{-1}$ is not injective into interval $(0,1)$ and $s 0$, since $f_{3}^{-1}$ has be the inverse function of $f_{3}$, we have contradiction.

Also, if $f((0, \infty)) \subset(0,1)$, then in the case of function $f_{4}:=f \mid(0, \infty)$ wo obtain a similar contradiction. 
From the abovo consideration the lanme follows.

is $\theta \mathrm{ma}$ 1. If $P$ is a continuous solution of (1)

for $k \neq 1$, then none of cases I, II and IV may exist.

Thus it is sufficjent to consider the case III. At first

we shall prove the following lema.

I e $\mathrm{m}$ a 2. If $f$ is a continuous solition of tha equation (1) for $k \neq I$ and satisfies the case III, $p$ being constant in $(9)$, than for every $x \in(-|p|, 0)$ there exists $z \in(x, 0)$ such that $f(z)>f(x)$.

proof. Let $x \in(-|p|, 0)$. Te will calculate

$$
\lim _{y \rightarrow \infty}\left(x f^{k}(y)+y f^{l}(x)\right)
$$

For this purpose we consicier tine guotient $\frac{f^{k}(y)}{y}$. From the form of the equation ( 1 ) and from the fact that $f_{1}$ is strictly Increasing it follows that lim $f(g)=\infty$. Mureover, beciuse tho equation ( 9 ) is true, hence for $g>0$ the function $f$ la differentiable at a point $y$. From de I'Hospltal's theorem and from of inverse furction theoren for the function (9), wo have

$$
\begin{aligned}
\lim _{y \rightarrow-\infty} \frac{f^{k}(y)}{y} & =\lim _{y \rightarrow \infty} k f^{k-1}(y) f^{\prime}(y)=\lim _{y \rightarrow \infty} \frac{k f^{k-1}(y)}{p\left(k f^{k-1}(y)-1 f^{1-1}(y)\right)}= \\
& =\lim _{y \rightarrow \infty} \frac{1}{p-\frac{p I}{k} f^{1-k}(y)}=\left\{\begin{array}{l}
0, \text { for } k<1, \\
\frac{1}{p}, \text { for } k>1 .
\end{array}\right.
\end{aligned}
$$

Hence,

$$
\begin{aligned}
& \underset{y \rightarrow \infty}{\lim }\left(x f^{k}(y)+y f^{l}(x)\right)=\lim _{y \rightarrow \infty} y\left(x \frac{f^{k}(y)}{g}+f^{l}(x)\right)=
\end{aligned}
$$

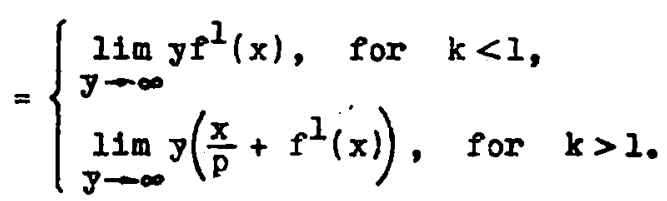


Thus

$\lim _{y \rightarrow \infty}\left(x f^{k}(y)+g f^{1}(x)\right)=\infty$, becauge $f^{l}(x)>1$ and $\frac{x}{p} \in(-1,0)$.

From (5) and the above equation it follows that there exisis $\bar{y}>0$ such that $x<x f^{\bar{k}}(\bar{y})+\bar{y} f^{l}(x)<0$.

Taking $z:=x f^{k}(\bar{y})+\bar{y} f^{l}(x)$ from (3) wo obtain $f(y)>f(x)$, Where $x<z<0$, which was to be proved.

Lemma 2 contradicts the fact that arbitrary continuous solution of the equation (1) satiofying the case III is strictiy decreasing in interval $(-\infty, 0)$. Thus we have the following le mma.

I $e$ o $a$ 3. For $k \neq I$ the equation (1) has not continuous solutions setisfying the case III.

Prom Coroilaxy, Lemma 1 and Lemme 3 we obtain the theorem.

Th o r a 2. The equation (1) for $k \neq i$, in the class of continuous functions $f: R-R_{0}$, has the unigue solution $f(x) \equiv 1$.

3. Remarks

a) When $k=0$ and $I=1$, from (1) we oitain (2). Its sointion in the class of continuous functione $f: R \rightarrow R$ was published in [2]. Among the continuous funotions satiafying the equetion (2) only $f(x) \equiv 1$ aseunes values from $R_{0}$. Let us notice that restriction of the range of the function $f$ is naturgl (ase [2] or [9]).

b) From the theorem.2 it follows that in the theorem 2 from $[\gamma]$ the assumption $" \bar{\alpha}_{2} \neq 0$ and $\bar{\alpha}_{3}=0$ exiot such that

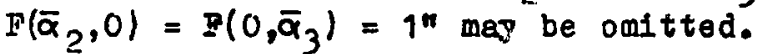

c) Knowledge of solutione of the equation (1) 1s important ot determining subsemigroups of the group $I_{n}^{1}(n>2)$ such that first paremeter depende oal ngmaining $n-1$ parameters (see [7]). 


\section{RBFERE TCES}

[1] J.G. D h o m b 5 : Solution générale sur un groupe Ebélien da l'équation fonctionnelle $f\left(x^{*} f(y)\right)=f\left(f(x)^{*} y\right)$, Aequationes Math. 15 (1977) 173-193.

[2] S. G o $1 \mathrm{a}$, A. $S \circ \mathrm{b} 1 \mathrm{~b}=1.1$ Sur l'équation fonctionnalie $f(x+y)(x !)=f(x) f(y)$, Publ. Math. Debrecen 6 (1959! 113-125.

[3]P. J a $v$ o : On the general aclution of the functional equation $f(x+y f(x))=f(x) f(y)$, Aequationes Math. 1 (1968) 235-238.

[4] S. M i a u r a : Sur la détermination de certains sous-grolipes du groupe $\mathrm{I}_{a}^{1}$ à l'aide d'équstions fonotionnelles, Dissertationes Math. 105 (1973).

[5] S. Ih i d a r a : sur les solucions de l'équation fonctionnelle $\varphi\left(\alpha \varphi(\beta)+\beta \varphi^{k}(\alpha)\right)=\varphi(\alpha) \varphi(\beta)$. Secosó International Symposium on Functional: Equationo and Inegualities. Debrecen, Hurgarg 1979.

[5] S. Mi dura, B. N s w a : c rozwiązanjach rówrania funkcyjnego $\varphi\left(\alpha \varphi(\beta)+\beta \varphi^{k}(\alpha)\right)=\varphi(\alpha) \varphi$ i $\left.\beta\right)$, Rocznik Naukowo-Dydaktyczn WSP w Kzeszowie 5/41 (1979) 59-68.

[7] S. i 1 d $r a, F . U r$ b a : 0 rozwiqzaniach równania finkcyirnego $\varphi\left(\alpha \varphi^{k}(\beta)+\beta \varphi^{l}(\alpha)\right)=\varphi(\alpha) \varphi(\beta)$, Rocznik Naukowo-Iydaktyc\%ns WSP Rzeszowie 6/50 (1982) 93-99.

[8] C.Gh. P o p a : Sur l'équation fonctionnelle $f(x+y f(x))=f(x) f(y)$, Ann. Polon. Math. 17 (1965) 193-i98.

[9] S. Wol o d z k o : Solution fénéraie de l'équation fonctionnelle $f(x+y f(x))=f(x) f(y)$, Aequationes Hath. 2. (1968) 12-29.

INSTITUTE CF MATHELATICS, FEDAGOGICAL UNIVERSITY, 35-310| RZESZ OW, POLAND

Recoived ilay 31, 1982. 
